\title{
Clinical Profile of Extremity Vascular Surgeries at Trivandrum Medical College
}

\author{
Sony Paul Suraja ${ }^{1}$, Vinu C. V. ${ }^{2}$, Kishore Lal J. ${ }^{3}$, Ravi Kumar T.D. ${ }^{4}$, Suresh Kumar J. ${ }^{5}$ \\ 1,2,3,4,5 Department of Cardiovascular \& Thoracic Surgery, Government Medical College, \\ Thiruvananthapuram, Kerala, India.
}

\begin{abstract}
\section{BACKGROUND}

One of the most common emergency diseases is traumatic vascular injury. The damage mechanism is linked to the environment and the time frame. The treatment of vascular injury is evolving in tandem with the passage of time. In short, vascular injury treatment ranges from simple to complex, and from simple to diverse. Patients suffering from vascular injury can now benefit from advanced medical equipment. Traditional treatments, on the other hand, continue to play an important role. It remains to be seen which treatment method will be adopted as the standard. Traumatic vascular injury is an emergency in surgery, mainly characterized by damage to veins and arteries. Haemorrhage (commonly from truncal vascular injuries) and ischemia (typical of peripheral arterial injuries) are the main clinical manifestations of vascular injuries. If vascular trauma has not been treated in time, it may cause disability or even death, especially for limb vascular surgery. There are many difficulties in diagnosis and treatment of vascular injuries, which have brought many challenges to the surgeons since the $20^{\text {th }}$ century. Vascular trauma accounts for $3 \%$ of all traumatic injuries. Vascular injury may lead to severe complications, so early diagnosis and timely treatment are critical. The purpose of this study was to assess the clinical profile of emergency extremity vascular cases at Trivandrum medical college.
\end{abstract}

\section{METHODS}

The demographic details, aetiology, mode of injury, limb and structures involved, concomitant injuries, clinical finding, delay in reaching trauma centre and surgical intervention done were recorded in a planned proforma and analysis was done. In this retrospective cross-sectional study, a total of 67 patients with extremity vascular emergencies referred to and called for intraoperative assistance in cardiovascular and thoracic surgery, Trivandrum Medical College over a period of 2 years from January 2018 to December 2019were included.

\section{RESULTS}

The mean age was 44 (SD 16.2) years with male to female ratio being $10.1: 1.0$. Trauma was the most common aetiology of vascular emergency (76.1\%). Out of the total study population, lower extremity emergencies (82 \%) were high compared to upper extremity emergencies (18\%). Popliteal and superficial femoral arteries were the most common sites of injury in lower extremity, whereas radial and brachial arteries were the most common arteries involved in the upper extremity. Vessels were seen contused in majority of patients $(46.3 \%)$ followed by thrombosis, transection and rupture. No patients presented with venous injuries. But tendon and nerve injuries were present in 36 patients (53.7 \%). Average time delay in reaching trauma center was 3 hours. Simple injuries to arteries were repaired with simple stitches as direct repair in 2 patients $(2.99 \%)$ and end to end anastomosis was done in 19 patients after resecting the injured segment.

\section{CONCLUSIONS}

Majority of the affected patients belonged to younger age group with trauma being the predominant aetiology. This may be due to the increase in incidence of road traffic accidents and other industrial accidents. Road safety measures are needed and awareness among public should be increased to reduce these types of injuries. All patients presented to our centre could be managed by performing emergency vascular surgeries, owing to timely reporting to hospital and by better use of imaging modalities.

\section{KEY WORDS}

Extremity Vascular Surgery, Anastomosis, Embolectomy
Corresponding Author:

Dr. Vinu C. V.,

Pranavam TC5/33,

Chembakavilasam Road, Sreekaryam P.O, Thiruvananthapuram, Kerala, India.

E-mail:drvinucv@gmail.com

\section{DOI: $10.14260 / j e m d s / 2021 / 652$}

How to Cite This Article:

Suraja SP, Vinu CV, Lal JK, et al. Clinical profile of extremity vascular surgeries at Trivandrum medical college. J Evolution Med Dent Sci 2021;10(37):3207-3212, DOI: $10.14260 /$ jemds/2021/652

Submission 25-03-2021, Peer Review 29-05-2021, Acceptance 05-06-2021, Published 13-09-2021.

Copyright (c) 2021 Sony Paul Suraja et al. This is an open access article distributed under Creative Commons Attribution License [Attribution 4.0 International (CC BY 4.0)] 


\section{BACKGROUND}

Trauma is the primary aetiology of vascular injury, which is a leading cause of death and disability in society. Despite the development of fully fledged trauma systems to enhance the delivery of quick and effective care, as well as numerous technical breakthroughs with improved clinical results and greater use of data collecting systems, the societal overload of traumatic injuries keeps rising. Sadly, there has been a steady rise in the number of trauma-related fatalities over time. Certainly, trauma mortality is complicated, with outcomes based on the extent and severity of suffered injuries. Nonetheless, haemorrhages are the leading cause of mortality in the vast majority of cases.

While our capacity to identify and treat vascular injuries has greatly increased over time, our overall influence on the incidence and frequency of these injuries has been limited. This bleak reality highlights the complexities of traumatic injury as well as the numerous factors that impact its prevalence in society. Although applied systematically to many disease processes affecting public health, an epidemiological methodology to the classification and treatment of traumatic vascular damage has received very little attention.

An epidemiological perspective to vascular damage offers numerous potential advantages, particularly in the context of civilian trauma. At the national level, understanding the many socioeconomic, geopolitical, and cultural variables that influence trauma can serve as a foundation for wide public health programmes, policy reform, and other activities targeted at reducing the impact of trauma on vulnerable people.

Furthermore, the application of epidemiologic fundamentals to vascular injury seeks to more precisely categorize and risk stratify multiple injury patterns through better data collection and scoring methodologies, to identify local and regional disparities in terms of volume as well as case mix, and to keep an eye on the changes with time. The goal of this study is to give a perspective for assessing the complex variables that impact vascular damage in a community from an epidemiological perspective.

Vascular surgeries accounts for one of the major emergencies performed worldwide. The aetiology of emergency vascular surgeries are multifactorial. It can be neck, chest, abdomen and emergency vascular surgeries which includes vascular trauma, acute arterial occlusion, ruptured peripheral and aortic aneurysms or aortic dissection. Among this, vascular trauma constitutes 2 - $3 \%$ of all trauma cases.

Although the recent developments in imaging modalities have led to the improvement in management of patients with vascular emergencies, in developing countries, the timely management of vascular emergencies depends upon various factors like pre-hospital care, referral to trauma care centre and early surgical intervention. The main goals in management of patients with extremity vascular injuries are first, saving the life of the patient and second, to save the affected extremity thus preventing limb loss.

The objective of this study was to identify the characteristics of patients presenting to the emergency department with extremity vascular emergencies in a tertiary care centre in south Kerala - Trivandrum Medical College.

\section{METHODS}

In this retrospective study, we reviewed medical records of 67 patients referred to and called for intraoperative assistance in cardiovascular and thoracic surgery, Trivandrum Medical College over a period of 2 years from January 2018 to December 2019. Informed consent was taken from the patients while preparing the detailed proforma and ethical committee clearance was also taken.

We included all cases during this period. In this study, vascular emergency cases except redo vascular surgeries and patients who underwent amputation owing to nonsalvageable injuries were included in the study. The vascular trauma cases were primarily assessed clinically by vascular, plastic and orthopaedic surgeons to assess the extent of injury and the possibility of salvaging the limb. Diagnosis was mostly made by physical examination. Simultaneous resuscitation and assessment was performed. Recordings of mode of injury, associated trauma and time since injury was also made. In cases of vascular injury with soft signsdecreased distal pulses, non-pulsatile and non-expanding hematoma, imaging studies were used. Vascular cases like acute arterial occlusions and multilevel vascular injuries were subjected to imaging-duplex ultrasound/angiogram for better localization of site of pathology. Vascular injuries were managed in emergency operating room under general anaesthesia. Patients were given intravenous fluids and preoperative preparation with cross matching of blood group, urinary catheterization etc. were done. Vascular injuries were repaired prior to other injuries i.e. bone, nerve and tendon injury.

Emergency vascular interventions like embolectomy, ligation, direct repair, repair with venous interposition graft, and very rarely with poly tetra fluoro ethylene (PTFE) graft etc. were performed depending on the aetiology. The suture used was polypropylene. Prophylactic fasciotomy was performed in patients with longer duration of ischemia for preventing compartment syndrome. Even before revascularization, fasciotomy was performed in patients who had tense muscle compartment in legs. Associated orthopaedic injury was managed by reduction of fracture and immobilization. Flap cover and nerve repair if needed was done by plastic surgeons.

The vascular repair integrity was confirmed once again after the fixation of bone, any stretch or laxity of the anastomosed vessel was corrected and closure of wound was done. Post-operatively, all patients were given anticoagulation, intravenous antibiotics and if needed blood transfusion was also done. Intensive monitoring was done post operatively to identify the success of vascular integrity and for reperfusion injury. Systemic and local signs were monitored to rule out reperfusion injury. Acute kidney injury and myoglobinuria were the systemic parameters monitored. Local feature suspected was the compartment syndrome. After assessing the limb salvage rate, patients were discharged with antiplatelets (ecospirin or clopidogrel).

The data extraction sheet included information on age, sex, aetiology, mode of injury, limb and structures involved, concomitant injuries, clinical finding. Delay in reaching trauma centre and surgical intervention was done. 


\section{Statistical Analysis}

Categorical and quantitative variables were expressed as frequency (percentage) and mean +/- SD respectively. Statistical analysis was performed using a $\mathrm{R}$ statistical software.

\section{RESULTS}

\begin{tabular}{|c|c|c|c|}
\hline \multicolumn{2}{|c|}{ Variable } & $\mathbf{n}$ & $\%$ \\
\hline & Mean age (years) & \multicolumn{2}{|c|}{44} \\
\hline \multirow{2}{*}{ Gender } & Male & 61 & 91 \\
\hline & Female & 6 & 9 \\
\hline \multirow{3}{*}{$\begin{array}{l}\text { Mechanism of vascular } \\
\text { emergency }\end{array}$} & Trauma & 51 & 76.1 \\
\hline & Thrombus / Emboli & 13 & 19.4 \\
\hline & AVF rupture & 3 & 4.48 \\
\hline \multirow{2}{*}{ Localization of injury } & Upper extremity & 12 & 18 \\
\hline & Lower extremity & 55 & 82 \\
\hline & Table 1. Patient Pr & & \\
\hline
\end{tabular}

A total of 67 patients who underwent various vascular interventions were included in the study. This study comprised of $61(91 \%)$ men and $6(9 \%)$ women with a mean age of 44 years who underwent emergency vascular interventions (Table 1). Average delay in presenting to hospital was 3 hours.

Trauma was the most common aetiology of vascular emergency $(76.1 \%)$. Next commonest aetiology was acute thrombosis/embolism followed by AV fistula rupture. Out of the total study population, lower extremity emergencies $(82$ $\%$ ) were high compared to upper extremity (18\%)

\begin{tabular}{|ccc|}
\hline Clinical Presentation & n & $\%$ \\
Haemorrhage & 20 & 29.9 \\
Extremity ischemia & 47 & 70.1 \\
\hline \multicolumn{2}{c}{ Table 2. Clinical Presentation of Patients } \\
\hline
\end{tabular}

Extremity ischemia in the form of loss of pulse was seen in 47 patients whereas active bleeding was seen in 20 patients $(29.9 \%)$

\begin{tabular}{|cccc|}
\hline & Affected Vessel & n & $\%$ \\
Upper extremity & Radial artery & 1 & 1.49 \\
& Brachial artery & 11 & 16.4 \\
\multirow{2}{*}{ Lower extremity } & Popliteal artery & 30 & 44.8 \\
& Superficial femoral artery & 25 & 37.3 \\
\hline \multicolumn{2}{|c|}{ Table 3. Vascular Injuries According to Localization } \\
\hline
\end{tabular}

Popliteal (44.8\%) and superficial femoral arteries (37.3 $\%)$ were the most common sites of injury in lower extremity, whereas radial $(1.49 \%)$ and brachial arteries $(16.4 \%)$ were the most common arteries involved in the upper extremity.

\begin{tabular}{|ccc|}
\hline Condition of Vessel & n & $\%$ \\
Contusion & 31 & 46.3 \\
Rupture & 3 & 4.48 \\
Thrombosis & 14 & 20.9 \\
Partial transection & 13 & 19.4 \\
Complete transection & 6 & 8.96 \\
\hline \multicolumn{2}{c}{} \\
\hline
\end{tabular}

Vessels were seen contused in majority of patients (46.3 \%) followed by thrombosis, transection and rupture. No patients presented with venous injuries. But tendon and nerve injuries were present in 36 patients (53.7 \%)

Simple injuries to arteries were repaired with simple stitches as direct repair in 2 patients (2.99\%) and end to end anastomosis was done in 19 patients after resecting the injured segment. (Table 5). In 31 patients (46.3\%), interposition venous grafting was done. Simple ligation was used for small vessel injuries in one patient. Embolectomy was performed in 13 patients $(19.4 \%)$.

\begin{tabular}{|ccc|}
\hline Technique & n & \% \\
\hline Direct repair & 2 & 2.99 \\
Embolectomy & 13 & 19.4 \\
End to end anastomosis & 19 & 28.4 \\
Ligation & 1 & 1.49 \\
Vein graft transposition & 31 & 46.3 \\
AVF disconnection / Brachial artery repair & 1 & 1.49 \\
\hline Table 5. Surgical Procedures Performed \\
\hline
\end{tabular}

\section{DISCUSSION}

The major cause of amputation of limb is extremity vascular injuries, if not identified and treated early. Penetrating injuries mostly affect the vascular supply at a single level whereas gunshot injuries and blunt trauma can affect the blood supply at multiple levels. The more extensive the vascular injury and the injury to the surrounding structures, the more complicated the management is, as the main and collateral vascular supply could be severely interrupted. The manifestation of vascular injury is mainly ischemia or bleeding or both, resulting in limb loss or mortality as per the severity of the injury. The clinical condition of the patient at presentation determines the initial assessment and the diagnostic techniques to be performed. Bleeding is the most common presenting symptom which can lead to hypovolemic shock. ${ }^{1}$ The primary aim of the surgeon is to prevent the exsanguination and death followed by restoration of vascularity to the extremity for limb salvage. ${ }^{1}$ Even the imaging studies can be avoided in such conditions where the limb is threatened or the life of the patient is in danger. Nihan Kayalar et al. ${ }^{2}$ observed that absence of imaging does not usually pose any challenge in detecting the vessel injury site. Exploration of injury site reveals the vessels affected. This can be done without any delay even in the absence of imaging studies. When there is difficulty in identifying the site of injury through the entry site, the incision can be extended proximally or distally and thorough exploration of the vessel can be done. Therefore, longitudinal incisions are preferred in extremity injuries and can be modified intraoperatively.

This study comprises 61 (91\%) men and 6 (9\%) women with a mean age of 44 years who underwent emergency vascular intervention. Average delay in presenting to hospital was 3 hours. In a similar study in North India by Somasekhar and Arunanshu et al. 132 patients ( $89 \%$ ) were males and 16 (11\%) were females. The mean age of the study population was 39 years. This is consistent with our present study. But the median delay in reaching the casualty department was 9.3 hours after the injury. But in our study, this delay was reduced as the transportation facilities and referral system in health sector in South Kerala is much advanced when compared to the North Indian states. Topal et al. also reported that majority of patients were males (95\%), female patients comprised only of $5 \%$ in the study. The large number of cases were seen in young men. This can be explained as they are more subjected to accidents and injuries as they are at the peak of activities.

In our present study, 20 patients (29.9\%) presented with active bleeding whereas other studies give a different picture. In a study by Kayaler et al. ${ }^{2} 74.5 \%$ presented with active 
bleeding, but majority of patients $(70.1 \%)$ presented with extremity ischemia in our study. European literatures and trauma registries showing pattern of distribution of vessels injured reveals higher number of lower limb vessel injuries compared to upper limb vessels. This is consistent with the present study.

When ischemia is the presenting symptom, diagnostic studies are usually performed to localize the site of vascular damage. But we should be careful to minimize the ischemic time for preventing the extremity loss. There is a suggestion that ankle-brachial pressure index (ABPI) should be the initial first line of choice to exclude the arterial injury. ${ }^{3}$ Another easily available and cost-effective investigation is the operator dependent, doppler ultrasound. But sometimes it is technically difficult to perform in open wounds with soft tissue loss and in patients with severe pain. ${ }^{4}$ Angiography is the best investigation available for localization of the site of vascular damage. It is time consuming as it requires transferring the patient to the imaging room. CT angiography is currently accepted as the first line imaging modality as it is non-invasive, rapid and accurate in detecting the vascular lesions. ${ }^{5,6}$ The CT angiography can be used post-operatively also to identify graft failure in patients with persistent or recurrent ischemia. It is the duty of the attending surgeon to confirm that time had not been wasted in performing these imaging studies, as the chance of survival of limb decreases with delay in diagnosis.

The presence of concomitant venous injuries is a little difficult, in patients with extensive tissue loss which disables the use of doppler study. Venous injuries are mostly identified intra operatively, causing bleeding during the procedure, which can make the vascular exploration a bit difficult. Concomitant venous repair for the salvation of affected extremity was emphasized by Ustunsoy et al. ${ }^{7}$ and Tunerir et al. ${ }^{8}$ Intra operatively, thorough exploration is needed to assess the status of veins, if missed, the injuries to major veins can lead to venous gangrene resulting in limb loss. However, the management of venous injuries remains controversial. Some advocate venous ligation whereas some insisted on primary venous repair or repair with synthetic graft material. In a study by Timberlake et al. no permanent sequelae was observed after ligation of veins in $70 \%$ of 322 venous injuries.

Nerve and tendon injuries can also be a part of extremity trauma. Prompt identification of these injuries are important for the repair. Concomitant soft tissue and bony injuries pose a major challenge in extremity vascular injuries especially the lower extremity injuries. Extensive soft tissue disruption and bony loss can increase the chance of amputation. Also, vascular injuries at multiple levels and more than one artery can also be associated with high chance of limb loss.

There are different surgical techniques for restoration of vascularity to the affected limb: Simple direct repair, Bypass grafting, end to end anastomosis after resection of affected vessel. Yet another option is the ligation of injured vessel. But it is not practiced now as it results in higher rates of amputation. ${ }^{9}$ It is done only for injuries of small sized veins or small side branches of arteries. Partial laceration of vessel can be repaired directly. When it is not possible, other option is end-to-end anastomosis after resection of the ends of affected vessel. Adequate mobilisation of arteries should be done before anastomosis, otherwise it would lead to stretching of anastomotic site. In cases requiring vascular conduit, autologous vein graft or PTFE/Dacron graft can be used. Autologous vein is harvested from opposite limb to prevent venous stasis of the extremity involved in injury. It is easily available, has superior patency rate and it resists infection. Bypass grafting of distal arteries is opted in case of large tissue defect, multiple bone fractures and higher infection risk. ${ }^{2}$ The bypassed graft may be passed in a tunnel created in unaffected area in order to protect the graft. Embolectomy can be performed when there is suspicion of presence of thrombus in proximal or distal part of involved vessel, before its repair is completed. Adequate soft tissue coverage of vessel is needed for obtaining good results.

Endovascular techniques for the management of vessel injuries has emerged as an important option. ${ }^{10}$ Ideal candidate for endovascular procedures are in those with injuries in areas difficult to surgically expose and when there is increased risk of iatrogenic injuries. ${ }^{10}$ Its advantages are less operating time, less blood loss and less iatrogenic injuries. But it has high operating cost.11,12 Endovascular techniques are used to control bleeding in trauma: embolization, balloon occlusion, covered stent. Exclusion of arteriovenous fistulas and pseudoaneurysms can be done by either coil embolization or covered stent.10,13-16

Compartment syndrome can occur due to delay in restoring blood flow. It may be the result of micro circulation impairment which can lead to irreversible damage and ischemia to nerves and muscles. Generally, 4 to 6 hours is considered to be the crucial hours or golden hours in restoring blood supply as it is the accepted duration of ischemia tolerance of muscles. Other factors leading to compartment syndrome are presence of concomitant venous injuries, multiple arterial injury, multiple bone fractures and blood loss. Early prophylactic decompressive fasciotomy lowers the chance of neuromuscular damage. Superior results are observed when prophylactic fasciotomy is done rather than early therapeutic decompression. Reperfusion injury is yet another complication which can occur when delayed restoration of vascularity is achieved. Immediate fasciotomy, vascular shunts as surgical adjuncts, ischemic condition and regional limb cooling also reduce the ischemic injury.

All the patients presented in our setting had arterial injuries. None had concomitant venous injuries. But nerve and tendon injuries were seen in patients in our study. None had extensive soft tissue injury or vascular injuries at multiple levels. Study by Rasouli et al. ${ }^{17}$ and Kayalar et al. ${ }^{2}$ showed concomitant arterial, venous and nerve injuries. In a study by Feliciano et al. $63.9 \%$ had arterial and $36.1 \%$ had venous injuries. More than $50 \%$ of patients had both arterial and venous injuries. ${ }^{18}$ Similar results were seen in a study by Topal et al. venous injuries in $45 \%$ cases, bone fracture 31.4 $\%$ and nerve injury seen in $16.4 \%$ patients.

The present study showed that popliteal (44.8\%) and superficial femoral arteries (37.3\%) were the most common sites of injury in lower extremity, whereas radial (1.49\%) and brachial arteries (16.4\%) were the most common arteries involved in the upper extremity. In a similar study by Gopinathan and Santhanam et al. the most common injured vessel was popliteal artery (64\%), the next being femoral artery (28\%). But the salvageability of femoral artery injury was higher than injury to popliteal artery. Another study by Kohli et al. showed that femoral vessels were the most 
common damaged vessel in the lower limb whereas brachial artery was commonly injured upper limb vessel.

In our study, $2.99 \%$ underwent direct repair, $1.49 \%$ ligation, $46.3 \%$ repair with interposition venous graft, 28.4 $\%$ end to end anastomosis, $19.4 \%$ embolectomy and $1.49 \%$ arteriovenous fistula (AVF) disconnection and brachial artery repair. Feliciano et al. ${ }^{18}$ in their study had $28.4 \%$ patients with end-to-end anastomosis and $38.8 \%$ patients with venous interposition graft whereas in a study in North India by Menakuru et al. ${ }^{19}$ repair without graft interposition was done in $74 \%$ and autogenous vein graft was used in $23 \%$ of cases. In another study by Humphrey et al. ${ }^{20} 51 \%$ underwent direct repair, $16 \%$ autogenous venous graft, $6 \%$ synthetic graft and $19 \%$ underwent ligation. We performed prophylactic fasciotomy in patients with longer ischemia duration.

\section{CONCLUSIONS}

This study was conducted at Trivandrum Medical College, a prestigious tertiary care centre in South India describing the patient profile who presented with extremity vascular emergencies. Majority of the affected patients belonged to younger age group with trauma being the predominant aetiology. This may be due to the increase in incidence of road traffic accidents and other industrial accidents. Road safety measures are needed and awareness among public should be increased to reduce these types of injuries. All patients presented to our centre could be managed by performing emergency vascular surgeries, owing to timely reporting to hospital and by better use of imaging modalities. This shows the effectiveness of prompt transportation facilities and the efficiency of referral system in health sector in Kerala. If delay in transfer occurs, prophylactic fasciotomy should be performed even in peripheral centres if compartment syndrome is suspected. Training in duplex scan if given to all vascular surgeons, can reduce the angiographic delay and thus early decision for vascular exploration and repair can be done in cases where ambiguity exists. However, endovascular procedures were not attempted in those patients due to lack of availability of infrastructure on emergency basis. The less delay in reaching tertiary care centre, team approach, early revascularization and postoperative care helps to achieve functional limb salvage. The small sample size and duration of study were the limitations in the current study.

Data sharing statement provided by the authors is available with the full text of this article at jemds.com.

Financial or other competing interests: None.

Disclosure forms provided by the authors are available with the full text of this article at jemds.com.

\section{REFERENCES}

[1] Nitecki SS, Karram T, Ofer A, et al. Management of combat vascular injuries using modern imaging: are we getting better? Emergency Medicine International 2013;2013:689473.
[2] Kayalar N, Boyacıoğlu K, Ketenciler S, et al. Emergency vascular injuries: patient profile, management strategies and risk factors for mortality. Turk Gogus Kalp Dama 2017;25:74-81.

[3] Kurtoğlu M, Dolay K, Karamustafaoğlu B, et al. The role of the ankle brachial pressure index in the diagnosis of peripheral arterial injury. Turkish Journal of Trauma and Emergency Surgery 2009;15(5):448-52.

[4] Meissner M, Paun M, Johansen K. Duplex scanning for arterial trauma. The American Journal of Surgery 1991;161(5):552-5.

[5] Genovese EA, Fonio P, Floridi C, et al. Abdominal vascular emergencies: US and CT assessment. Critical Ultrasound Journal 2013;5(Suppl 1):S10.

[6] Poveda TS, Uribe CH, Vásquez RC, et al. Efficacy of multidetector computed angiotomography in the diagnosis of limb arterial injuries. Cirugía Española (English Edition) 2013;91(4):257-62.

[7] Ustunsoy H, Tuncozgir B, Sanli M, et al. Peripheral vascular injuries. Turk Gogus Kalp Dama 1999;7:140-3.

[8] Tünerir B, Beşoğul Y, Yavuz T, et al. Peripheral artery injury and results of treatment. GKDC Dergisi 1998;6(2):151-4.

[9] Yahya MM, Mwipatayi BP, Abbas M, et al. Popliteal artery injury: royal perth experience and literature review. ANZ Journal of Surgery 2005;75(10):882-6.

[10] Johnson CA. Endovascular management of peripheral vascular trauma. Seminars in Interventional Radiology 2010;27(1):38-43.

[11] Reuben BC, Whitten MG, Sarfati M, et al. Increasing use of endovascular therapy in acute arterial injuries: analysis of the National Trauma Data Bank. Journal of Vascular Surgery 2007;46(6):1222-6.

[12] Du Toit DF, Coolen D, Lambrechts A, et al. The endovascular management of penetrating carotid artery injuries: long - term follow - up. European Journal of Vascular and Endovascular Surgery 2009;38(3):267-72.

[13] Lopera JE. Embolization in trauma: principles and techniques. Seminars in Interventional Radiology 2010;27(1):14-28.

[14] Irahara T, Sato N, Moroe Y, et al. Retrospective study of the effectiveness of Intra - Aortic Balloon Occlusion (IABO) for traumatic haemorrhagic shock. World Journal of Emergency Surgery 2015;10(1):1.

[15] Naughton PA, Park MS, Morasch MD, et al. Emergent repair of acute thoracic aortic catastrophes: a comparative analysis. Archives of Surgery 2012;147(3):243-9.

[16] Farber A, Tan TW, Hamburg NM, et al. Early fasciotomy in patients with extremity vascular injury is associated with decreased risk of adverse limb outcomes: a review of the National Trauma Data Bank. Injury 2012;43(9):1486-91.

[17] Rasouli MR, Moini M, Khaji A. Civilian traumatic vascular injuries of the upper extremity: report of the Iranian national trauma project. Ann Thorac Cardiovasc Surg 2009;15(6):389-93.

[18] Feliciano DV, Herskowitz KE, O'Gorman RB, et al. Management of vascular injuries in the lower extremities. The Journal of Trauma 1988;28(3):319-28. 
[19] Menakuru SR, Behera A, Jindal R, et al. Extremity vascular trauma in civilian population: a seven - year review from North India. Injury 2005;36(3):400-6.
[20] Humphrey PW, Nichols WK, Silver D. Rural vascular trauma: a twenty - year review. Annals of Vascular Surgery 1994;8(2):179-85. 